International Journal of

Advanced Science and Convergence

\title{
Wireless Sensor Network Development: Explosion Locator Using Artificial Neural Network
}

\author{
Leah A. Alindayo ${ }^{1}$, and Marven E. Jabian² \\ ${ }^{1}$ Department of Computer Engineering, St. Peter's College, Philippines \\ ${ }^{2}$ Department of Electrical Engineering, Mindanao State University-Iligan Institute of Technology, Philippines
}

\begin{abstract}
Background/Objectives: This paper think about the huge advancement in the innovation of sensor of remote interchanges and remote sensor applications. The created framework that identify and passes on the impacts blast area that will push the power to reaction right away. Methods/Statistical analysis: In this paper, improvement of remote sensor arrange (think about the three sensors) were painstakingly put in an area to effectively assemble information. The information accumulated from these three sensors were fed to artificial neural network (ANN) for preparing. The remote sensor arrange framework is equipped for gathering, putting away and breaking down information from a few devices installed in the framework and the preparation the framework to recognize the impacts blast. Findings: Results show that the multi-sensor combination and coordination assumes a basic job in the general plan and execution upgrade of explosion locator being created. Improvements/Applications: The fit is about perfect for train, looking at and traverse 0.9999 the entire reaction of precision by means of percent error with the most extreme 0.067731 has been accomplished.
\end{abstract}

\section{Index Terms}

ANN, Computer Applications, Computer Engineering, Levenberg-Marquart, Wireless Sensor Networks.

\footnotetext{
Corresponding author : L.A. Alindayo

Leah_alex@yahoo.com

- Manuscript received April 17, 2020.

- Revised May 18, 2020 ; Accepted June 20, 2020.

- Date of publication June 30, 2020.

(C) The Academic Society of Convergence Science Inc.

2619-8150 ( 2019 IJASC. Personal use is permitted, but republication/redistribution requires IJASC permission.
} 


\section{INTRODUCTION}

The development of Wireless Sensor Network (WSN) technology has applied in monitoring the natural disasters by augmenting a combination of sensors as low cost and quick response. Explosion Locator is a system that detects and conveys the location of an explosion or other weapon fired using the combination of sensors. The uses of sensor network using JZ863 mini Power Wireless sensor to achieve the research objectives. The use of frequency network systems allows the achievement of tracking the location of explosion. As such, it effectively leads to the reduction of injuries and casualties in homeland security operations, and primarily been used in dispatch centers for quick reaction of explosion incidents.

The design systems have a great advantage since it can be deployed in a vast and even in remote and low accessibility areas. The sensor network can also function unattended and its configuration be done remotely. The use of sensor networks bring down the cost of system maintenance and data acquisition since the means of collecting data can be done automatically. Deployment of sensor networks may represent a substantially more economical method for conducting this study.

The structure frameworks have an incredible favorable position since it very well may be conveyed in an immense and even in remote and low openness regions. The sensor system can likewise work unattended and its design be done remotely. The utilization of sensor systems cut down the expense of framework upkeep and information obtaining since the methods for gathering information should be possible naturally. Sending of sensor systems may speak to a considerably increasingly prudent strategy for directing this investigation.

The created framework is fit for gathering, putting away, and examining the information from a few remote sensor gadgets. Blast area frameworks for the most part require additionally detecting modalities to distinguish the blast. This framework that utilization acoustic-just strategies are characterized as those frameworks with detecting modalities focused at acoustic marvels. This framework helps experts in finding the blast area at incorporated level. Essentially, it expands the reaction productivity of police association or any exploring body. Moreover, this framework helps in controlling the wounds and setbacks in country security tasks.

\section{OBJECTIVES}

This study is to design and fabricate a device to detect the explosion location employing artificial neural network which can help authorities to response efficiently. The following are the specific objectives:
1. To create an architectural design for the proposed system.

2. To design a sound sensor circuit for explosion detection.

3. To design the network of sensor for a specific area of choice.

4. To deploy the device to a certain area and gather results that will be fed to an ANN for training.

5. To determine the efficacy of the system through actual field test.

\section{OVERALL PLATFORM}

The framework configuration (see Figure 1) comprises of the Network Section and Receiver Section. The planned framework acoustically recognize blast and transmit that data through a system of transmitters and collectors associated with the ANN framework. The blast areas are transmitted and gathered which is bolstered to PC and the blast data is shown on an electronic guide which recognizes property limits of the area.

\section{A. Network Section}

The Network Section show the equipment structure which include a transmitter and a beneficiary

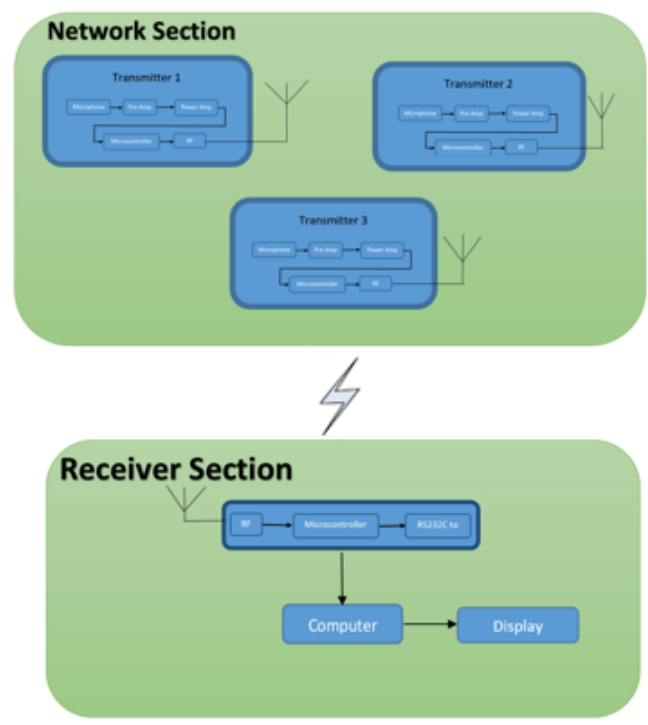

Figure 1. Wireless Sensor Network Design

framework. This plan is utilized to recognize gag impacts blasts and transmit the sound by methods for a collector framework and that will be sustained to ANN and the structure of the firmware.

The transmitter configuration as showed up in Figure 1 is used to transmit acoustic sign through a receiver. A recipient changes over the weight waves realized by a sound into vibrates inside a twist which changes the vibrations into electric signal. 
Preamplifiers increase a recipient sign to line-level (for instance the level of sign quality required by such devices) by giving a consistent addition while envisioning provoked upheaval that would somehow mangle the sign.

A preamplifier (preamp) is an electronic enhancer that prepares a little electrical sign for further strengthening or taking care of. A preamplifier is routinely put close to the sensor to diminish the effects of upheaval and impedance. It is used to heighten the sign from the receiver.

The sign goes out from control speaker is changed over to cutting edge by the ADC module of the microcontroller and transmit the sign through RF Technology as demonstrated Figure 3 is used as the remote data transmission in short detachment.

From the beginning of the structure of transmitter and the collector framework, the scientist downloaded an example blast sound from the web that will fill in as the contribution to the microcontroller. As the example tried, it discovered that the plan dependent on figure 3 and figure 5 was accomplished. In this way, the analyst finished the circuit plan, the last circuit design. The following stage is to secure the materials required for the usage and gadget development. As the usage is done, the analyst lead the testing and as appeared in information and result the genuine creation of the transmitters TX1, TX2, TX3 and the gatherer gadget.

\section{B. Receiver Section}

The recipient configuration as appeared in Figure 2 gets information from the transmitter the collector system. configuration as appeared in Figure 2 gets the gathered information from the transmitters

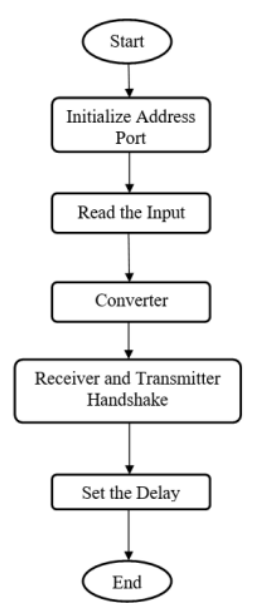

Figure 2 Process Flow Chart of Transmitter

that will be nourished to ANN framework.

The get signal is as pixel from 0 to 1023 . In accepting the transmitted sign, the scientist planned the framework where every one of the transmitters is given a $10 \mathrm{msec}$ to transmit the sign into the recipient segment. Utilizing the RS232C to associate with the $\mathrm{PC}$ to store the get sign to the information lumberjack.

\section{Firmware Design}

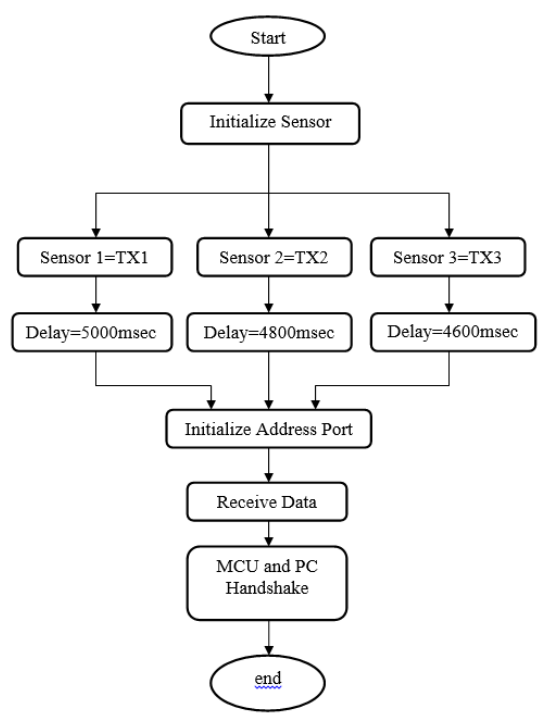

Figure 3 Process Flow Chart of Receiver

Figure 3 on the below shows the process flow chart of the transmitter section. It is a process in the implementation of program for the transmitter and the linking of receiver and transmitter as well.

Figure 3 shows the procedure stream diagram of the collector segment. It is a procedure in the usage of the collector area and the connecting among recipient and the $\mathrm{PC}$.

\section{Actual Setup}

Figure 4 shows the real arrangement of the framework at Camp Thomas, Tipanoy, Iligan City. The reference purpose of the arrangement is situated at $\mathrm{TX} 1(0,0)$.

E.

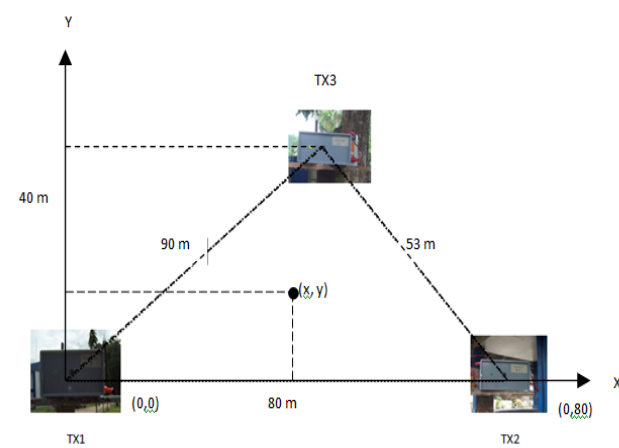

thi

an

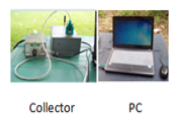

th

co 
example, the Microphone, the intensifier, the power enhancer, the microcontroller and the RF segment all together execute the transmitter device.

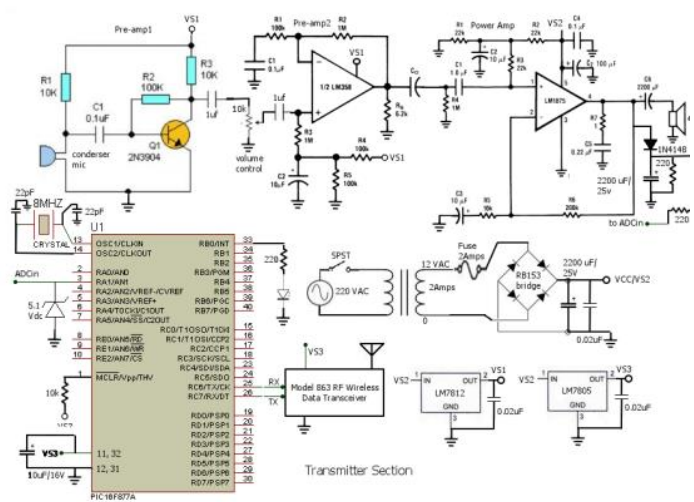

Figure. 5 Circuitry of a transmitter Design

Figure 6 shows the circuit format of the recipient plan. It incorporates the accompanying parts, for example, the RF, microcontroller, UART associated with PC.

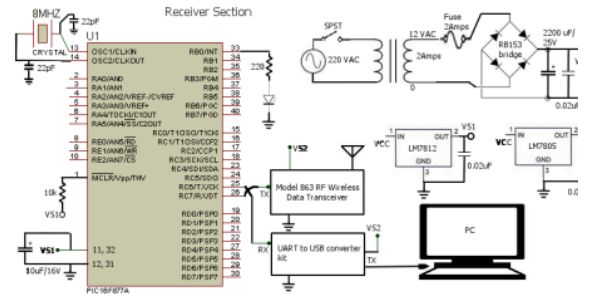

Figure 6 Circuitry of a Receiver Design

\section{F. Data Gathering}

So as to assemble the information, the framework is sent at camp Thomas, Tipanoy, Iligan City. As appeared in Figure 7 beneath transmitters are situated in three corners of the territory of inclusion considered. The white banners speak to the genuine

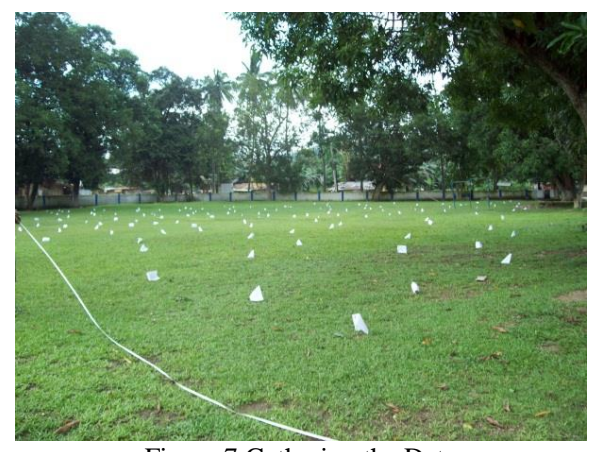

Figure 7 Gathering the Data

blast of the directed shotgun sparkler that speaks to an information.
Table 1 underneath shows the accumulated information during the arrangement of framework at Camp. Thomas, Iligan City, which is the $20 \%$ of 140 assembled information utilized for testing the prepared system.

\section{Table 1 SAMPLE Untrained Data}

\begin{tabular}{|c|c|c|c|c|c|c|}
\hline \multicolumn{3}{|c|}{ Input } & \multicolumn{2}{c|}{ Target } & \multicolumn{2}{c|}{ Output } \\
\hline TX1 & TX2 & TX3 & X & Y & X & Y \\
\hline 236 & 355 & 283 & 35.97073 & 6.83643 & 35.9348 & 6.8296 \\
\hline 281 & 353 & 210 & 67.4702 & 6.690784 & 67.4028 & 6.6841 \\
\hline 297 & 359 & 430 & 67.23126 & 14.90299 & 67.1641 & 14.8881 \\
\hline 288 & 371 & 349 & 45.35301 & 17.33562 & 45.3077 & 17.3183 \\
\hline 282 & 402 & 351 & 48.49675 & 11.30169 & 48.4483 & 11.2904 \\
\hline 236 & 392 & 299 & 19.83051 & 13.19668 & 19.8107 & 13.1835 \\
\hline 315 & 350 & 357 & 34.46463 & 13.04113 & 34.4302 & 13.0281 \\
\hline 203 & 370 & 245 & 35.19586 & 8.760151 & 35.1607 & 8.7514 \\
\hline 318 & 352 & 355 & 37.67123 & 14.3129 & 37.6336 & 14.2986 \\
\hline 246 & 352 & 315 & 28.33941 & 7.904096 & 28.3111 & 7.8962 \\
\hline
\end{tabular}

Figure 8 shows the approval and test information window. It shows the determination segment of the first dataset that will be utilized for the approval and testing the default estimations of $15 \%$. In this investigation consider the $70 \%$ dataset for preparing the ANN.

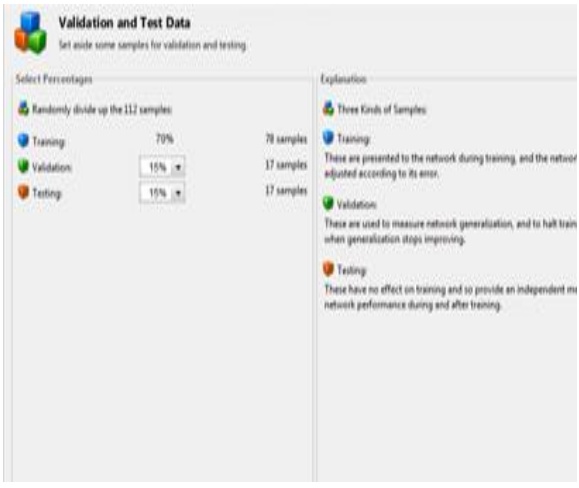

Figure 8 Neural Network Fitting tool (nftool)

Figure 9 shows the feed-forward backpropagation utilizing the "trainlm" engineering which called a Levenberg-Marquart feed-forward back-propagation. It is preparing fuction that updates the loads and the predispositions as per the improvement utilized. It is significant that the loads are ajusted so as to make the real yields near the objective or the deliberate yields of the system.

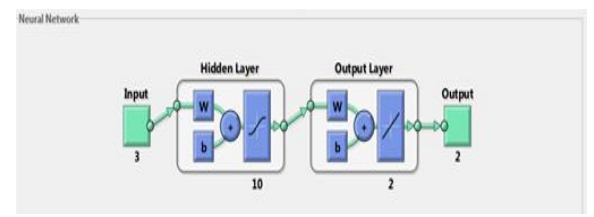

Figure 9 System Network 
While a system is prepared, Matlab naturally produces the presentation bend, relapse bend, and the cross approval error and tests mistakes. Figure 10 shows the presentation plot of approval errors and tests mistakes.

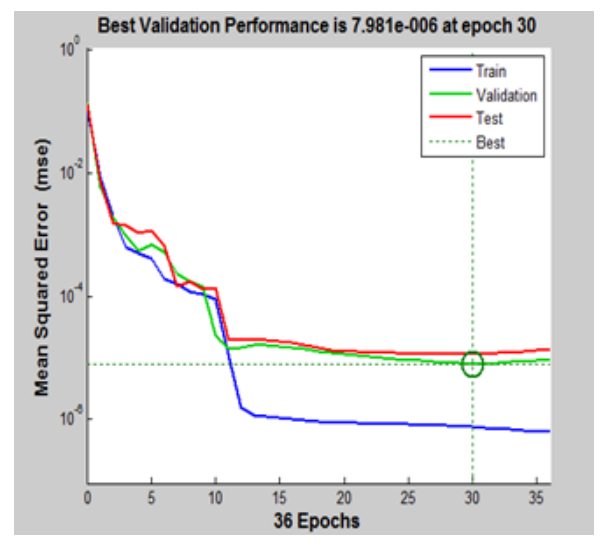

Figure 10 Neural Network Training Performance

Figure 11 shows the outcomes, the preparation stooped when the approval mistake and test error expanded for 30 emphasess, which happens at emphasis (or age) is 36 . The last mean-square mistake is little though the test errorand the approval set blunder have practically comparative charateristics (green and red lines). No noteworthy over-fitting has happened by cycle of 30 where the best approval happens.

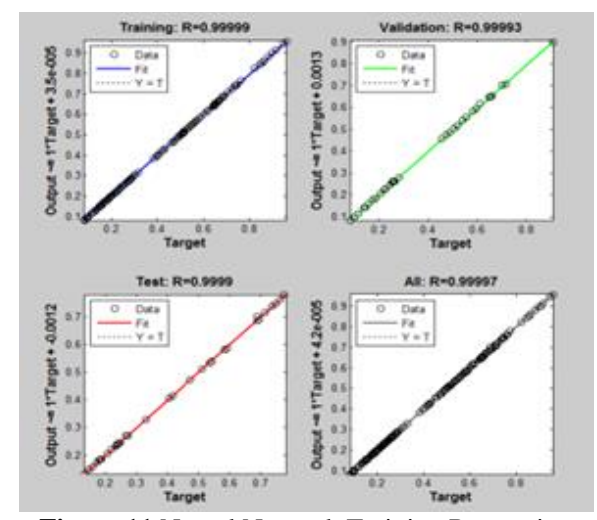

Figure 11 Neural Network Training Regression Graph

Figure 12 shows the GUI of personnel monitoring system with a sample. The red foundation shows that the framework gets a sign that there was a blast. In the window, it shows the transmitted sign at TX yield and it is put away in the information lumberjack. The arrange focuses and the relating area will be shown in the guide. It utilizes Matlab 2013.

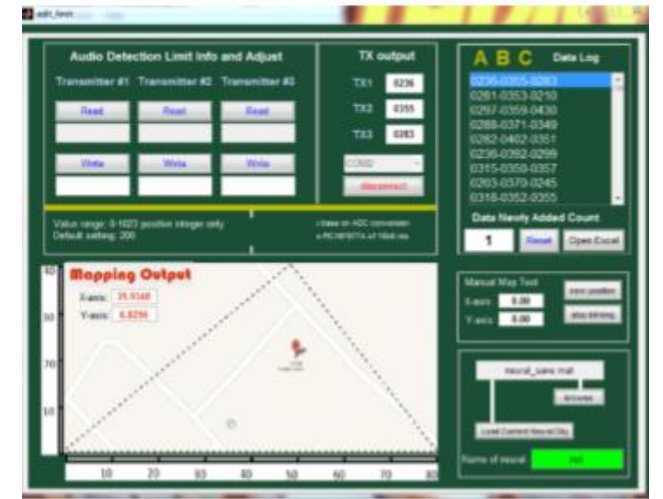

Figure 12 GUI Personal Monitoring System

\section{CONCLUSION}

The conceptualization and usage of the remote sensor organize for blast locator utilizing artificial neural network advancement were figured it out. The multi-combination and incorporation for correspondence between the two segments were effectively cultivated in the general plan and execution upgrade of the blast locator being created. In this investigation, Matlab instruments are utilized to find the precise area of blast. Out of 140 gathered information, $112(80 \%)$ information are utilized for preparing and $20 \%$ concealed information are utilized for testing. The result outcomes affirm the prevalence of feed-forward back-propagation with trainlm design with a low MSE values. In addition, ANN models have in great execution with the precision of $\%$ mistake yield is 0.067731 .

\section{ACKNOWLEDGMENT}

I would like to express my sincere gratitude to my adviser Marven Jabian,MS for providing their invaluable guidance, comments and suggestions throughout the study.

Also I would like to thank my family for the financial assistance for the implementation of this study.

\section{REFERENCES}

[1] Coman, C. I.,I. C.Vizitiu, and S. Demeter. (2004). Small arms fire tracking with an array of doppler sensors. In Radar Conference, 2004. EURAD. First European (pp. 25-28). IEEE. Retrieved on August 8, 2012 from http://goo.gl/R5JI8.

[2] Dingley, G., and C. Alabaster (2012). Radar based automatic target system, In Waveform Diversity and Design Conference, 2009 International (pp. 22-25). IEEE. Retrieved on from http://goo.gl/5E3SUF 
Haykin, S.,Neural Networks and Learning Manhines, Pearson Education, Inc. New Jersey, $3^{\text {rd }} \mathrm{Ed}$.

[3] Li, J. and Stoica,P., Mimo (2008). Radar Signal Processing, Honokin, NJ:Wiley

[4] Showen, R.L., Calhoun, Chu, W.C. and Dunham, J.W (2008). Acoustic gunshot location in complex environments:concepts and results. InSPIE Defense and Security Symposium.

[5] Repository Of Machine Learning Databases (2012). Retrieved on December 28, 2012 from ftp://ftp.ics.edu/pub/machinelearning-databases/.

[6] Sayed, A.H., Tarughat, A., and Khajehnouri, N. (2005). Network-Based Wireless Location, IEEE Signal Processing Magazine, Vol. 22

[7] Visa, A. Neural Computataion. Retrieved on December 28, 2012 from http:// www.cs.tut.fi/ avisa/2806nn1.ppt

[8] Wang, Q., Zheng, A.,Liu, X., and Sha, L (2008). Lightning: a hard real-time, fast and lightweight low-end wireless sensor election protocol for acoustic event localization. Mobile Computing, IEEE Transsactions.

[9] Zhang, Y.,M.Amin, and F. Ahmad (2007). A novel approach for multiple moving target localization using dual frequency radars and time-frequency distributions. In signals, systems and computers 